\title{
Aproximación a la patología presentada en las construcciones de tierra. Algunas recomendaciones de intervención
}

\author{
Approximation to the earth buildings pathology. \\ Some recommendations of intervention
}

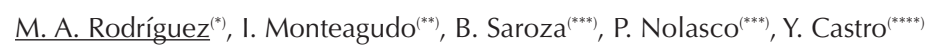

\section{RESUMEN}

En el presente artículo se ponen de manifiesto una serie de aspectos que es necesario tener presente a la hora de acometer un estudio sobre la patología presentada en las construcciones de tierra en general, y patrimoniales en particular.

Para ello, y tras proponer una serie de recomendaciones que contribuyan a un correcto diagnóstico de la patología presentada, se identifican las lesiones más características de este tipo de construcciones.

Por último, se proponen algunas pautas de intervención en función de la patología presentada, así como recomendaciones de carácter general para la conservación del patrimonio de estructuras de tierra.

113.104

Palabras clave: patrimonio arquitectónico, construcción con tierra, patología de la construcción, construcción sostenible, conservación de edificaciones.

\section{SUMMARY}

This article shows several points to consider in the study and conservation of the heritage of earth.

We show the most characteristic damages of these buildings and we propose some recommendations for the correct diagnostic of their pathology.

Finally, some recommendations for the conservation of this heritage are given.

Keywords: heritage building, construction of earth, earth buildings pathology, sustainable construction, building conservation.

\footnotetext{
(*) Universidad de Oviedo, Asturias (España)

${ }^{(* *)}$ Instituto Politécnico José Antonio Echeverría, Ciudad de la Habana (Cuba)

${ }^{(* * *)}$ Universidad Central de Las Villas, Santa Clara (Cuba)

${ }^{(* * *)}$ Centro Meteorológico Provincial de Villa Clara. CITMA, Santa Clara (Cuba)

Persona de contacto/Corresponding author: mangelrd@uniovi.es (M. A. Rodríguez)
}

Fecha de recepción: 26-02-09 Fecha de aceptación: 13-01-10 


\section{INTRODUCCIÓN Y OBJETIVOS}

El término arquitectura de tierra, adoptado en 1980 por la Conferencia Internacional sobre Construcciones con Tierra realizada en Ankara, Turquía, por su referencia al material natural empleado para conformar el hábitat a lo largo de la civilización de múltiples naciones y pueblos, puede considerarse novísimo en el léxico del ramo. No obstante, en el ámbito internacional se observa cierta bipolaridad en el manejo de esta arquitectura. Por una parte, la praxis de países desarrollados como Estados Unidos, Inglaterra, Alemania y Francia, que han asumido un gran desarrollo tecnológico con rigor científico-técnico probado tanto en el campo de la conservación como en la nueva construcción. En otras naciones, como son los países africanos y latinoamericanos, las soluciones de construcción con tierra tienen una profunda fundamentación cultural y social por la práctica "del hacer" trasmitida, en tanto las condiciones económicas no favorecen sustancialmente un desarrollo científico entorno a nuevas búsquedas y desempeños. Ante esta situación, puede afirmarse que el manejo global del tema demanda considerar ponderadamente las potencialidades tecnológicas que ofrece la tierra como material de construcción, y los valores culturales y arquitectónicos del producto resultante en un nivel intermedio de desarrollo económico.

La problemática general referida a la conservación del patrimonio histórico - arquitectónico - arqueológico construido con tierra, se aborda desde diversas disciplinas. Las investigaciones se han venido desarrollando con relación a la antropología y evolución tipológica de las estructuras, la preservación de sitios arqueológicos, la restauración arquitectónica, la patología constructiva, la metodología y técnicas de intervención, las investigaciones sobre la microestructura y composición química de la arcilla, la disminución del riesgo sísmico y otros desastres naturales, los casos prácticos, y las perspectivas tecnológicas y proyecciones futuras.

En Monteagudo (2001) (1) y en Dassler, L. et al. (1993) (2) se identifican los tipos de investigaciones, los objetos y objetivos de investigación más frecuentes, así como la demanda de estudios existentes.

El presente artículo tiene como objetivo general contribuir al conocimiento de las variables que intervienen en la investigación científica en el campo de la conservación del patrimonio y la edificación construida con tierra. Asimismo, como objetivos específicos se persigue proponer recomendaciones para un correcto diagnóstico de la patología presentada, identificar las lesiones más ca- racterísticas de estas estructuras y proponer intervenciones posibles en función de las lesiones presentadas.

\section{TRABAJOS REALIZADOS}

El conjunto de trabajos realizados ha seguido una metodología que ha ido de lo general a lo particular, matizada por fases de retroalimentación y reformulación en la medida que se ha ido avanzando. Del análisis crítico de los especialistas consultados y de las experiencias de campo desarrolladas por los autores a lo largo de varios años de interacción con las construcciones de tierra, fundamentalmente en Cuba, Monteagudo (1), Saroza (3) y Rodríguez et al. (4), se ha organizado el trabajo en las siguientes etapas:

I. Investigación preliminar. Tuvo por objetivo realizar un sondeo bibliográfico sobre la generalidad del tema, así como la realización de proyectos y entrenamientos técnicos de construcción con tierra en Cuba con diferentes alcances. Permitió a los autores dominar el campo general del conocimiento.

II. Planteamiento inicial. Comprendió la delimitación del problema de estudio, las hipótesis de investigación, los objetivos a alcanzar y los instrumentos de investigación científica a utilizar.

III. Profundización en el marco teórico de referencia. En esta fase se identificaron los conceptos y teorías necesarias para la fundamentación de las recomendaciones que se generan en el presente estudio.

IV. Estudio de campo. Para la caracterización y evaluación de las construcciones con tierra se desarrolló un estudio de campo que comprendió el 30\% de las edificaciones de tierra presentes en el Centro Histórico de La Habana, con distinto grado de protección y diferentes lesiones.

V. Análisis y discusión de los aspectos relativos a la tipología constructiva de tierra. En esta etapa se sistematizaron los resultados referentes a los tipos de edificaciones y sus partes componentes.

VI. Análisis y discusión de los aspectos relativos a la diagnosis de la patología característica de las construcciones con tierra. Al igual que la anterior permitió sistematizar y generalizar la patología característica de las construcciones con tierra estudiadas.

VII. Elaboración de las recomendaciones para la conservación del patrimonio en este campo. 


\section{RECOMENDACIONES PARA UN CORRECTO DIAGNÓSTICO}

Para el diagnóstico de la patología de las construcciones con tierra se suele aplicar una metodología de campo y otra de laboratorio, en el estudio de las causas, formas y mecanismos de alteración que han tenido lugar.

Como punto de partida para una diagnosis profunda que redunde en el diseño de subsiguientes tratamientos e intervenciones es imprescindible el conocimiento de las propiedades que presentan los distintos materiales de construcción y su respuesta ante los agentes y acciones exteriores.

Por otro lado, el estudio tanto de los materiales como de los elementos constructivos, se ha focalizado tradicionalmente en la tierra y en los morteros adyacentes por considerárseles los elementos esenciales en estas fábricas. Tomando como base los estudios realizados por CRATerre (5) y DGEMN (6), consideramos que el esfuerzo debe concentrarse en una diferenciación y diseño metodológico siguiendo las siguientes etapas:

I. Toma de datos preliminar, constatación y medición de los síntomas de alteración.

II. Monitoreo de la evolución de los síntomas.

III. Investigación de las causas.

IV. Investigación del origen de las causas.

V. Evaluación de los síntomas o indicadores de alteración mediante los métodos de ensayos y técnicas de diagnóstico y según la magnitud de los indicadores macroscópicos de alteración.

Las técnicas de diagnosis emplean ensayos tanto destructivos como no destructivos. Estudios e investigaciones más recientemente se basan en la estadística matemática mediante la creación de matriz de relaciones entre variables previamente determinadas. Ejemplo de esto último se pueden consultar en Monteagudo (1), Odul (7), Monjo et al.(8), Romeral et al.(9) y Chiari (10).

\section{PATOLOGÍA CARACTERÍSTICA DE LAS CONSTRUCCIONES DE TIERRA}

El análisis integral de procesos patológicos implica, en primer lugar, la identificación y entendimiento, tanto de los agentes patógenos internos (aquéllos intrínsecos al material) como de los agentes patógenos externos (definidos por agentes exteriores) que originan las causas de los deterioros visibles o indicadores macroscópicos de alteración.

\subsection{Factores intrínsecos}

Tan sólo en la literatura sobre geotecnia referente a la arquitectura y construcción de tierra, se pueden encontrar alusiones particulares sobre el comportamiento del material térreo ante los agentes ambientales. Por lo tanto, al estudiar las respuestas en el tiempo de la tierra puesta en edificaciones, ha de acudirse a la mecánica de suelos para comprender los fenómenos que originan las alteraciones y deterioros visibles. Son de suma importancia a la hora de estudiar la fábrica pétrea, factores intrínsecos como origen, composición, textura y estructura de la fase sólida del suelo constituyente de la obra. Por tanto, a la hora de estudiar el comportamiento del material en una construcción existente deben investigarse las características de la misma, el origen y comportamiento de la arcilla contenida, las propiedades fisicoquímicas y generales del suelo, así como la correspondencia de las mismas con el uso y procedimiento constructivo empleado.

\subsection{Factores extrínsecos}

Dentro de este conjunto de factores se engloban los agentes ambientales, los biológicos, los mecánicos y los antrópicos.

Dentro de los agentes ambientales podemos destacar, por su acción degradadora de la fábrica térrea y pétrea, los siguientes: agua, aire, temperatura, presencia de sales, contaminación atmosférica, fenómenos naturales.

Dentro de los agentes biológicos se engloba la biodegradación producida por insectos (abejas y termitas), animales (aves, roedores y domésticos) y plantas (superiores e inferiores).

El origen de los desórdenes estructurales que tienen su origen en factores mecánicos, pueden resumirse en cinco categorías: defectos del material, roturas mecánicas accidentales, mala concepción y diseño de las soluciones estructurales y constructivas y, por último, problemas y errores de ejecución en obra.

En cuanto a los factores antrópicos, pueden agruparse en tres categorías: diseño y planificación, producción y construcción, así como uso y explotación.

\subsection{Formas de alteración y deterioros}

Las reflexiones sobre los mecanismos de alteración más comunes a las construcciones de tierra permiten afirmar que el principal inconveniente es su rápida degradación ante la acción del intemperismo y los agentes ambientales. A diferencia de los eventos que suceden en las fábricas de piedra expuesta, las alteraciones en las obras de tierra se lo- 
calizan tanto en el elemento de terminación y revestimiento del paramento, como en el soporte, masa o estructura del mismo. En consecuencia, la diagnosis y determinación del estado patológico o alterado debe distinguir entre los fenómenos de la superficie y los de la estructura.

Con el objeto de identificar las alteraciones típicas de las construcciones con tierra, se han confeccionado las tablas 1 y 2 . En ellas, y tomando como punto de partida las investigaciones llevadas a cabo por Houben y Guillaud (11), se describen los desórdenes estructurales. Asimismo, se recogen las lesiones de la estructura asociadas a cada tipo de desorden. Hay que tener presente que las lesiones son los síntomas aparentes, mientras que la patología implica el origen del fenómeno o proceso patológico, que no siempre es visible.
Teniendo en cuanta lo que se acaba de comentar, se han elaborado las tablas 3 y 4, donde se han identificado y agrupado los factores intrínsecos y extrínsecos respectivamente, que pueden influir en el grado de alterabilidad de la fábrica térrea.

Como se puede ver, en la tabla 3 se identifican las características de la fase sólida del suelo que influyen en el uso y explotación del material tierra una vez puesto en obra. Éstas son la presencia o no de materia orgánica, tipos de minerales, tipos de arcillas constituyentes y fuerzas de enlace propias, propiedades y características generales, así como la correspondencia entre estas propiedades y el uso de la técnica de construcción aplicada en la obra.

Del mismo modo, en la tabla 4 se explicita el conjunto de factores extrínsecos, agrupados

Tabla 1

Desórdenes estructurales típicos en las construcciones con tierra y lesiones que se generan

\begin{tabular}{|c|c|c|c|c|}
\hline & \multicolumn{2}{|r|}{ Proceso patológico } & \multicolumn{2}{|r|}{ Lesiones que se originan } \\
\hline & \multirow{2}{*}{1.} & \multirow[b]{2}{*}{$\begin{array}{l}\text { Fallo por asentamiento y variaciones } \\
\text { del estrato de apoyo }\end{array}$} & - & Grietas inclinadas \\
\hline & & & $\bullet$ & $\begin{array}{l}\text { Desprendimiento del revestimiento y } \\
\text { soporte del muro }\end{array}$ \\
\hline & \multirow{4}{*}{2.} & \multirow{4}{*}{$\begin{array}{l}\text { Deterioro del basamento por ataque } \\
\text { de plagas, presencia de plantas, } \\
\text { accidentes, etc. }\end{array}$} & $\bullet$ & Acanaladuras y oquedades en el muro \\
\hline & & & $\bullet$ & $\begin{array}{l}\text { Grietas horizontales, verticales o } \\
\text { inclinadas }\end{array}$ \\
\hline & & & $\bullet$ & Fisuras lineales o radiales \\
\hline & & & $\bullet$ & $\begin{array}{l}\text { Desprendimientos de los } \\
\text { revestimientos o soporte del muro }\end{array}$ \\
\hline & 3. & Deterioro por mala ejecución de la & & $\begin{array}{l}\text { Grietas horizontales en juntas } \\
\text { verticales }\end{array}$ \\
\hline & & albañilería del soporte y los & $\bullet$ & Alabeos \\
\hline & & revestimientos & • & $\begin{array}{l}\text { Desprendimiento del material de } \\
\text { revestimiento }\end{array}$ \\
\hline \multirow{15}{*}{$\begin{array}{l}\text { Desórdenes } \\
\text { estructurales }\end{array}$} & \multirow{5}{*}{4.} & \multirow{5}{*}{ Fallos en las aberturas } & $\bullet$ & Grietas verticales \\
\hline & & & $\bullet$ & Grietas inclinadas \\
\hline & & & $\bullet$ & Grietas horizontales \\
\hline & & & $\bullet$ & Desplazamiento de las piezas \\
\hline & & & $\bullet$ & Flechas en la viga de cerramiento \\
\hline & \multirow{3}{*}{5.} & \multirow{3}{*}{$\begin{array}{l}\text { Fallos de ejecución en la parte } \\
\text { superior del vano }\end{array}$} & $\bullet$ & Fisuras lineales y/o radiales \\
\hline & & & $\bullet$ & Grietas horizontales \\
\hline & & & $\bullet$ & $\begin{array}{l}\text { Abombamiento por compresión axial } \\
\text { excéntrica }\end{array}$ \\
\hline & \multirow[t]{2}{*}{6.} & \multirow{2}{*}{$\begin{array}{l}\text { Penetración insuficiente de las vigas } \\
\text { del sistema de techo o entrepiso }\end{array}$} & & $\begin{array}{l}\text { Grietas inclinadas en las fogonadura de } \\
\text { las vigas }\end{array}$ \\
\hline & & & • & $\begin{array}{l}\text { Desprendimientos de revestimiento } \\
\text { y/o soporte del muro }\end{array}$ \\
\hline & \multirow{3}{*}{7.} & \multirow{3}{*}{$\begin{array}{l}\text { Deficiente solución del apoyo de los } \\
\text { sistemas de techo }\end{array}$} & $\bullet$ & Grietas inclinadas \\
\hline & & & $\bullet$ & Grietas horizontales \\
\hline & & & • & $\begin{array}{l}\text { Desprendimiento del material de } \\
\text { soporte y revestimiento }\end{array}$ \\
\hline & \multirow[t]{2}{*}{8.} & \multirow{2}{*}{$\begin{array}{l}\text { Deficientes soluciones de evacuación } \\
\text { del agua de lluvia sobre muros y } \\
\text { techos }\end{array}$} & $\bullet$ & $\begin{array}{l}\text { Desprendimiento del material de } \\
\text { soporte y revestimiento }\end{array}$ \\
\hline & & & - & Roturas de las piezas de soporte \\
\hline
\end{tabular}


Tabla 2

Desórdenes por humedad típicos en las construcciones con tierra y lesiones que se generan

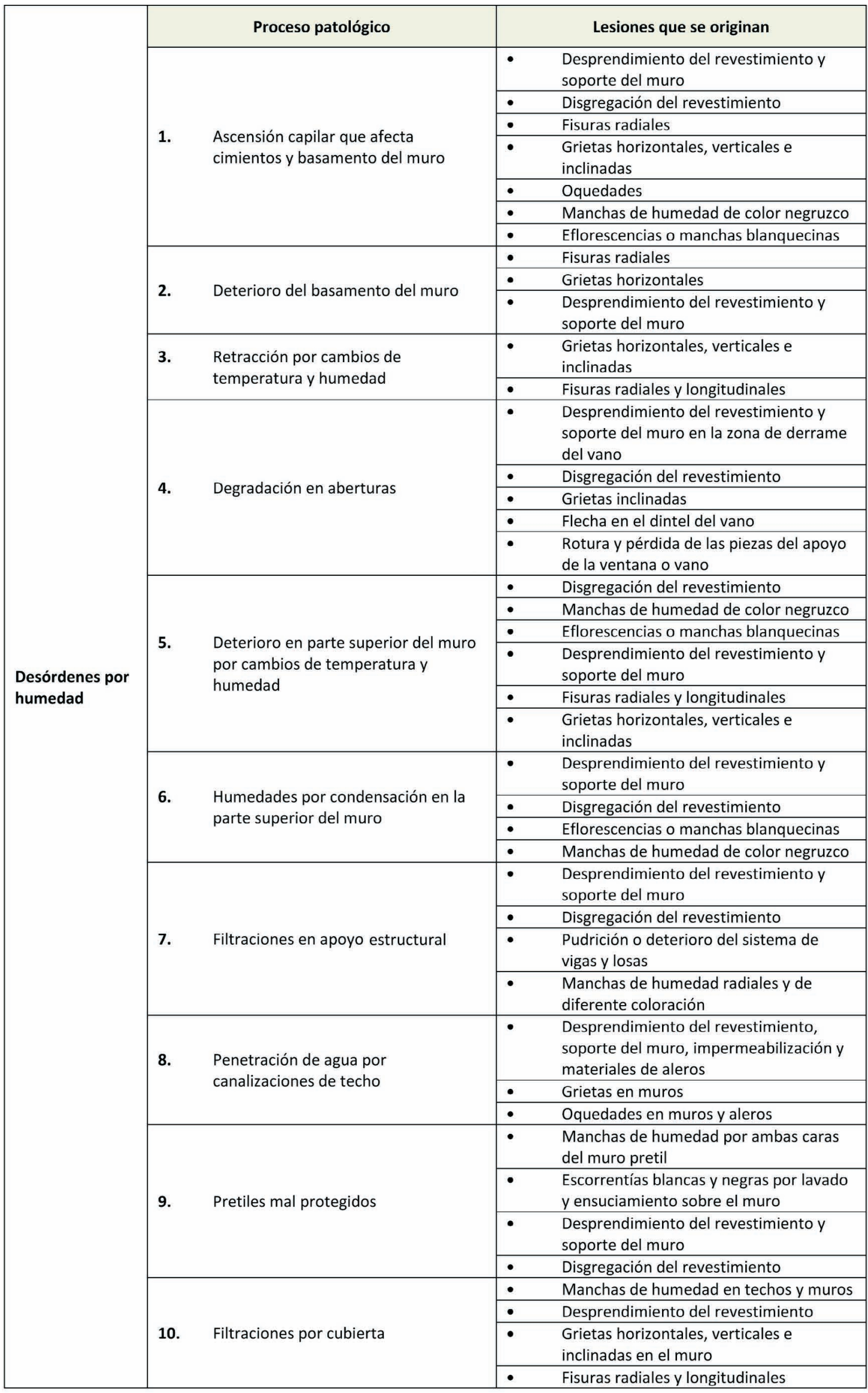


Tabla 3

Factores intrínsecos del material tierra

\begin{tabular}{|c|c|c|}
\hline \multicolumn{3}{|c|}{$\begin{array}{l}\text { Características de la fase sólida del suelo y otros aspectos intrínsecos que influyen en el uso y explotación del } \\
\text { material }\end{array}$} \\
\hline \multicolumn{3}{|c|}{ A. Carácter de la fase sólida del suelo } \\
\hline - Materia orgánica & & - Minerales \\
\hline \multicolumn{3}{|c|}{ B. Origen y estructura de la arcilla } \\
\hline - Tipos de arcillas & & - $\quad$ Fuerzas de enlace \\
\hline \multicolumn{3}{|c|}{ C. Propiedades y características generales } \\
\hline \begin{tabular}{l|l} 
- Propiedades carácter & " \\
químico & \\
\end{tabular} & Propiedades carácter físico & - Propiedades fundamentales \\
\hline \multicolumn{3}{|c|}{ D. Relación entre las propiedades del material y el uso en la edificación } \\
\hline
\end{tabular}

Tabla 4

Factores extrínsecos de alteración en la fábrica térrea

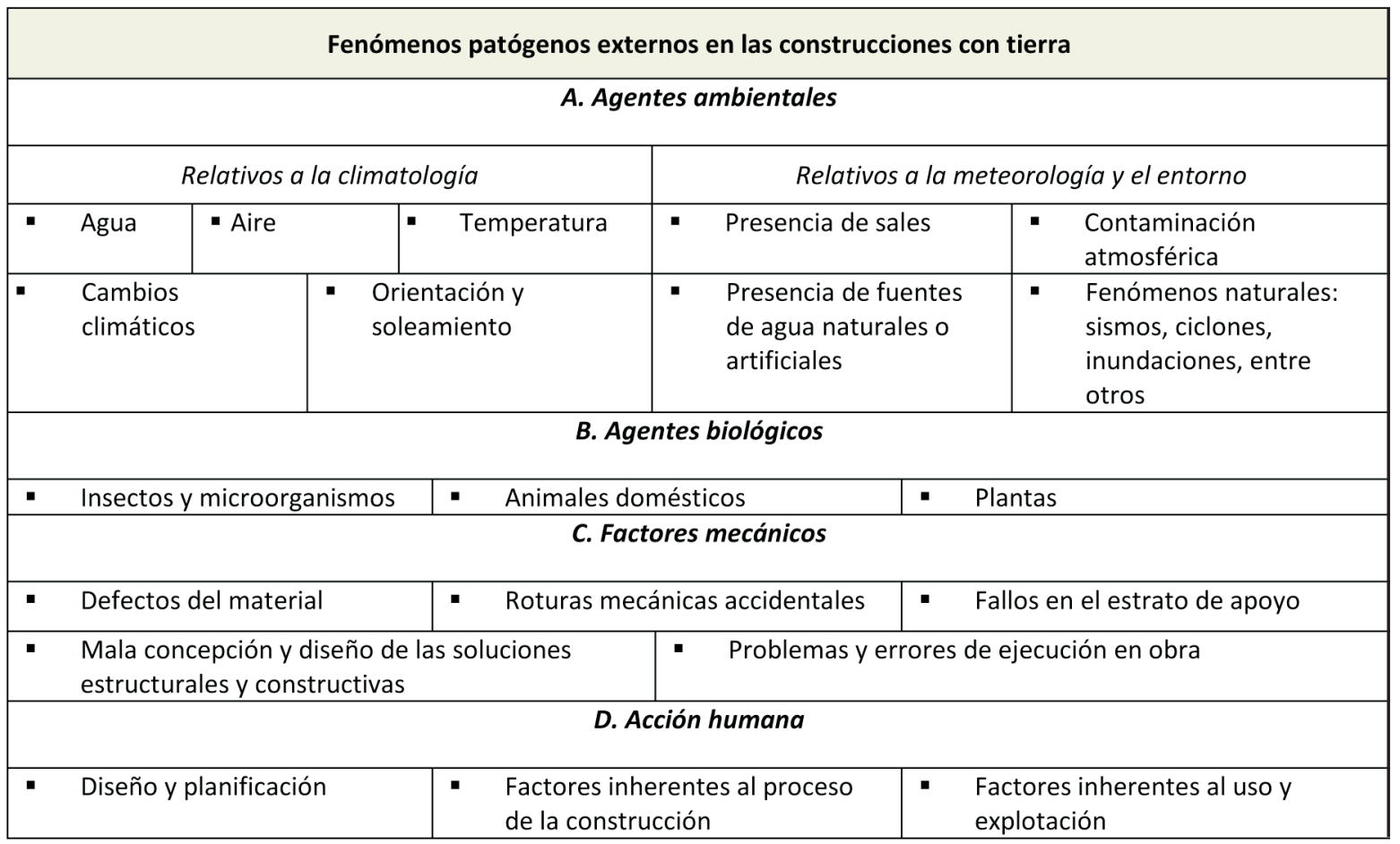

en agentes ambientales, agentes biológicos, factores mecánicos y factores antrópicos.

\section{TIPOLOGÍA DE INTERVENCIÓN EN FUNCIÓN DE LA PATOLOGÍA PRESENTADA}

La intervención en el patrimonio históricoarquitectónico de tierra no ha encontrado hasta el momento letra escrita en cartas, documentos o legislación alguna que regule o paute las acciones que se acometen con buenas intenciones, pero no siempre con buenos resultados. Se ha podido constatar en función de la experiencia de los autores en este tema, que cualquier intervención sobre una obra hecha con tierra se vuelve compleja por las tres razones siguientes:

- Generalmente la intención no es sólo consolidar o reparar, sino "poner en valor".

- No existen personas que dominen los oficios tradicionales, ni es fácil encontrar los materiales propios de aquellas técnicas.

- En muchas ocasiones, los materiales y procedimientos utilizados acarrean incompatibilidades o problemas añadidos a los que se tratan de solucionar. 


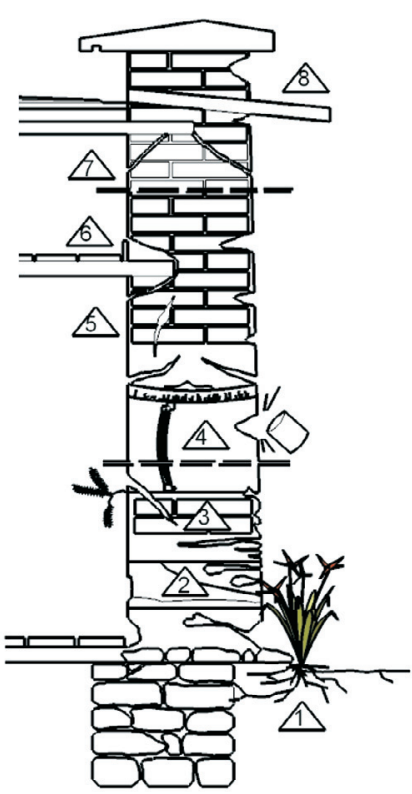

Lesiones típicas de la patología estructural

ICCRON (5) sintetiza en tres los criterios a tener en cuenta para lograr una viabilidad técnica y económica de la intervención. Estos criterios son los siguientes:

- Persistencia en el uso de técnicas de construcción tradicionales o contemporáneas, y nivel de satisfacción probado.

- Uso de materiales locales, apropiación de los mismos y costes de producción.

- Autenticidad y contemporaneidad del objeto de intervención, identificación y diferenciación de la intervención por diversas vías.

Del mismo modo, la ICCRON (5), DGEMN (6), Chiari (10) y Huben et al. (11), identifican las intervenciones posibles como las siguientes:

- Drenajes superficiales y profundos alrededor de las estructuras de barro.

- Tratamientos químicos de superficie y consolidación.

- Técnicas de inyección y sedimentación para la consolidación o impermeabilización.

- Estabilización estructural. Materiales y métodos de reforzamiento.

- Protecciones de bajo coste para atender los daños de erosión por lluvia o nieve derretida, que proporcione aislamiento térmico, pero a su vez, permeable al vapor de agua.

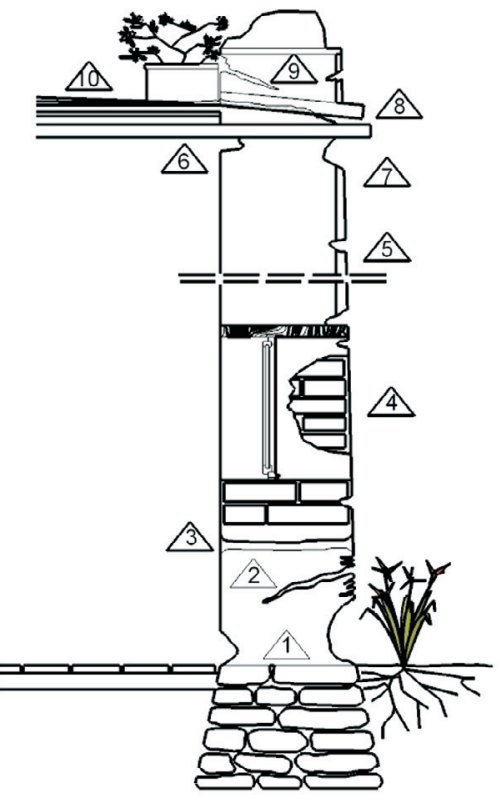

1. Patología típica de las construcciones con tierra.
Lesiones típicas de la patología por humedad

En cuanto a las tecnologías de preservación de adobe, Saroza (3) apunta lo siguiente:

- La preservación estará en función de la protección a las estructuras, de los elementos o agentes de deterioro y particularmente del agua.

- Se necesitan análisis que permitan unificar e identificar los agentes y factores de deterioro operantes en cada estructura y sitio.

- Es esencial la selección de materiales de preservación y metodologías de ensayos normalizados.

- Son de gran importancia la documentación y el monitoreo de los procesos de intervención para la preservación.

Teniendo en cuenta la anterior consulta bibliográfica, podemos resumir en las cuatro siguientes etapas las intervenciones más frecuentemente establecidas en relación con la conservación de sitios, elementos y estructuras construidas con tierra:
A. Reconstrucción - reintegración - refuerzo.
B. Estabilización - consolidación.

\section{Protección.}

D. Mantenimiento y conservación preventiva.

En la tabla 5 se presentan todos los aspectos que definen la patología de las construcciones con tierra, es decir, el nivel de deterioro, el indicador macroscópico de alteración y el agente de alteración. Para cada caso se 
Tabla 5

Síntesis de la relación entre indicador macroscópico de alteración, factores desencadenantes del deterioro y etapas de intervención recomendada

\begin{tabular}{|c|c|c|c|}
\hline $\begin{array}{l}\text { Nivel del } \\
\text { deterioro }\end{array}$ & $\begin{array}{l}\text { Indicador macroscópico } \\
\text { de alteración }\end{array}$ & Agente de alteración & $\begin{array}{l}\text { Etapas de intervención previa } \\
\text { eliminación de la causa y origen } \\
\text { del deterioro }\end{array}$ \\
\hline \multirow{6}{*}{ 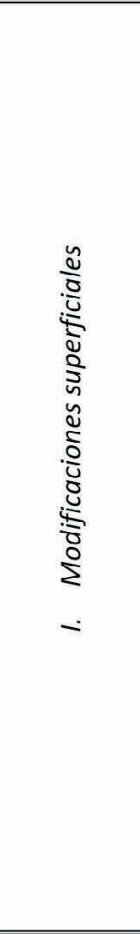 } & 1. Costra & $\begin{array}{l}\text { - Agua, agentes } \\
\text { contaminantes (polución), } \\
\text { agentes bióticos, etc. }\end{array}$ & $\begin{array}{l}\text { Limpieza } \\
\text { Protección superficial } \\
\text { Mantenimiento y } \\
\text { conservación preventiva }\end{array}$ \\
\hline & 2. Suciedad & $\begin{array}{l}\text { - Agua, agentes } \\
\text { contaminantes (polución), } \\
\text { viento }\end{array}$ & $\begin{array}{l}\text { Limpieza } \\
\text { Mantenimiento y } \\
\text { conservación preventiva }\end{array}$ \\
\hline & $\begin{array}{l}\text { 3. Manchas de } \\
\text { humedad }\end{array}$ & - Agua & $\begin{array}{l}\text { Limpieza } \\
\text { Protección de diverso tipo } \\
\text { Mantenimiento y } \\
\text { conservación preventiva }\end{array}$ \\
\hline & 4. Eflorescencia & - Agua, sales solubles & $\begin{array}{l}\text { Limpieza } \\
\text { Protección superficial y } \\
\text { drenaje } \\
\text { Mantenimiento y } \\
\text { conservación preventiva } \\
\end{array}$ \\
\hline & 5. Decoloración & $\begin{array}{l}\text { - Agua, sol, viento, agentes } \\
\text { contaminantes, sales } \\
\text { solubles, etc. }\end{array}$ & $\begin{array}{l}\text { Protección superficial y de } \\
\text { coberturas } \\
\text { Mantenimiento y } \\
\text { conservación preventiva }\end{array}$ \\
\hline & $\begin{array}{l}\text { 6. Desprendimiento de } \\
\text { pintura }\end{array}$ & - $\quad$ Agua, sales solubles & $\begin{array}{l}\text { Limpieza } \\
\text { Protección y consolidación } \\
\text { superficial } \\
\text { Mantenimiento y } \\
\text { conservación preventiva } \\
\end{array}$ \\
\hline \multirow{5}{*}{ 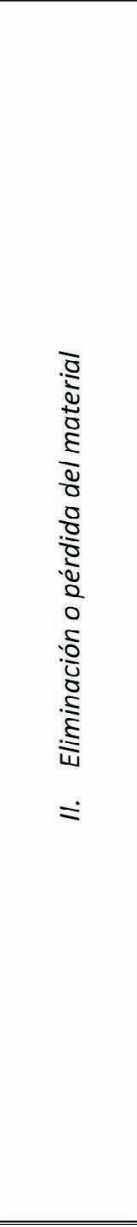 } & 7. Erosión & Acción eólica, agua & $\begin{array}{l}\text { Estabilización - consolidación } \\
\text { del soporte y el revestimiento } \\
\text { Reintegración material } \\
\text { Protección de diverso tipo } \\
\text { Mantenimiento y } \\
\text { conservación preventiva }\end{array}$ \\
\hline & 8. Disgregación & $\begin{array}{l}\text { Agua, temperatura, sol, } \\
\text { viento, sales solubles, } \\
\text { agentes bióticos, etc. }\end{array}$ & $\begin{array}{ll}\text { I. } & \text { Estabilización - consolidación } \\
& \text { del elemento disgregado } \\
\text { II. } & \text { Reintegración material } \\
\text { III. } & \text { Protección de diverso tipo } \\
\text { IV. } & \text { Mantenimiento y } \\
& \text { conservación preventiva } \\
\end{array}$ \\
\hline & $\begin{array}{l}\text { 9. Desprendimiento } \\
\text { del material del } \\
\text { muro }\end{array}$ & $\begin{array}{l}\text { - Agua, cambios térmicos y } \\
\text { climáticos, accidentes } \\
\text { naturales, vegetación } \\
\text { parásita, acción del hombre, } \\
\text { agentes mecánicos - físicos } \\
\text { intrínsecos a la estructura, } \\
\text { etc. }\end{array}$ & $\begin{array}{l}\text { Reconstrucción - } \\
\text { reintegración y refuerzo del } \\
\text { muro } \\
\text { Protección de diverso tipo } \\
\text { Mantenimiento y } \\
\text { conservación preventiva }\end{array}$ \\
\hline & $\begin{array}{l}\text { 10. Desprendimiento } \\
\text { del material del } \\
\text { revestimiento }\end{array}$ & $\begin{array}{l}\text { - Agua, cambios térmicos y } \\
\text { climáticos, accidentes } \\
\text { naturales, vegetación } \\
\text { parásita, acción del hombre, } \\
\text { agentes mecánicos - físicos } \\
\text { intrínsecos a la estructura, } \\
\text { etc. }\end{array}$ & $\begin{array}{l}\text { I. } \begin{array}{l}\text { Consolidación - reintegración } \\
\text { del revestimiento }\end{array} \\
\text { II. Protección de acuerdo a la } \\
\text { tipología del deterioro } \\
\text { III. Mantenimiento y conservación } \\
\text { preventiva }\end{array}$ \\
\hline & 11. Abofado & $\begin{array}{l}\text { - Agua, cambios térmicos y } \\
\text { climáticos, accidentes } \\
\text { naturales, vegetación } \\
\text { parásita, acción del hombre, } \\
\text { agentes mecánicos - físicos } \\
\text { intrínsecos a la estructura, } \\
\text { etc }\end{array}$ & $\begin{array}{l}\text { Previo desprendimiento del material } \\
\text { abofado, proceder ídem anterior }\end{array}$ \\
\hline
\end{tabular}




\begin{tabular}{|c|c|c|c|c|}
\hline \multirow{5}{*}{$\begin{array}{l}\tilde{y} \\
\frac{1}{3} \\
\frac{1}{3} \\
0 \\
\vdots \\
\vdots\end{array}$} & 12. Fisura & $\begin{array}{l}\text { - } \\
\text { moventes ambientales, } \\
\text { intrínsecos a la estructura o } \\
\text { al material, fenómenos } \\
\text { naturales, acción del } \\
\text { hombre, etc. }\end{array}$ & $\begin{array}{l}\text { I. } \\
\text { II. }\end{array}$ & $\begin{array}{l}\text { Consolidación - estabilización } \\
\text { del elemento fisurado } \\
\text { Mantenimiento y } \\
\text { conservación preventiva }\end{array}$ \\
\hline & 13. Grieta inclinada & $\begin{array}{l}\text { - Agentes ambientales, } \\
\text { movimientos y mecanismos } \\
\text { intrínsecos a la estructura o } \\
\text { al material, fenómenos } \\
\text { naturales, acción del } \\
\text { hombre, etc. }\end{array}$ & $\begin{array}{l}\text { I. } \\
\text { II. }\end{array}$ & $\begin{array}{l}\text { Reconstrucción - } \\
\text { reintegración - refuerzo } \\
\text { Mantenimiento y } \\
\text { conservación preventiva no } \\
\text { solo del muro de tierra, sino } \\
\text { también del resto de los } \\
\text { elementos estructurales de la } \\
\text { edificación }\end{array}$ \\
\hline & 14. Grieta horizontal & $\begin{array}{l}\text { - Agentes ambientales, } \\
\text { movimientos y mecanismos } \\
\text { intrínsecos a la estructura o } \\
\text { al material, fenómenos } \\
\text { naturales, acción del } \\
\text { hombre, etc. }\end{array}$ & & Ídem anterior \\
\hline & 15. Grieta vertical & $\begin{array}{l}\text { - Agentes ambientales, } \\
\text { movimientos y mecanismos } \\
\text { intrínsecos a la estructura o } \\
\text { al material, acción del } \\
\text { hombre, etc. }\end{array}$ & & Ídem anterior \\
\hline & $\begin{array}{c}\text { 16. Desplome o } \\
\text { vuelco }\end{array}$ & $\begin{array}{l}\text { - Agentes ambientales, } \\
\text { movimientos y mecanismos } \\
\text { intrínsecos a la estructura o } \\
\text { al material, fenómenos } \\
\text { naturales, acción del } \\
\text { hombre, etc. }\end{array}$ & $\begin{array}{l}\text { I. } \\
\text { II. } \\
\text { III. } \\
\text { IV. }\end{array}$ & $\begin{array}{l}\text { Reconstrucción - } \\
\text { reintegración - refuerzo del } \\
\text { elemento constructivo } \\
\text { Estabilización - consolidación } \\
\text { del material a reintegrar } \\
\text { Protección de diverso tipo } \\
\text { Mantenimiento y } \\
\text { conservación preventiva }\end{array}$ \\
\hline \multirow{2}{*}{ 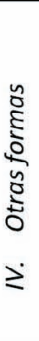 } & $\begin{array}{l}\text { 17. Modificaciones } \\
\text { antrópicas }\end{array}$ & - Hombres y mujeres & II. & $\begin{array}{l}\text { Reconstrucción y } \\
\text { reintegración de formas y } \\
\text { materiales originales o } \\
\text { coherentes } \\
\text { Mantenimiento constructivo }\end{array}$ \\
\hline & 18. Plantas superiores & - $\quad$ Agentes bióticos & $\begin{array}{l}\text { I. } \\
\text { II. } \\
\text { III. } \\
\text { IV. }\end{array}$ & $\begin{array}{l}\text { Limpieza } \\
\text { Consolidación } \\
\text { Protección superficial } \\
\text { Mantenimiento y } \\
\text { conservación preventiva }\end{array}$ \\
\hline
\end{tabular}

pueden observar las correspondientes recomendaciones de intervención en función de la patología presentada.

\section{RECOMENDACIONES GENERALES PARA LA CONSERVACIÓN DEL PATRIMONIO DE EDIFICACIONES DE TIERRA}

Con el fin de contribuir a la conservación del patrimonio de edificaciones térreas que han perdurado hasta nuestros días, proponemos las siguientes sugerencias o recomendaciones:

- Catalogación y documentación del fondo edificado y los bienes culturales, con énfasis en el inventario de técnicas y modos tradicionales de hacer.

- Diseñar y ejecutar procesos de intervención que prevean programas de mantenimiento y manejo adecuado de los objetos intervenidos.
- Experimentación de sistemas, materiales y tratamientos con énfasis en la durabilidad de las estructuras, con capacidad de respuesta ante los riesgos de desastres por accidentes y eventos de ocurrencia como ciclones, temporales de lluvia, inundaciones, sismos, etc.

- Implementación de programas para la capacitación y entrenamiento de especialistas.

- Establecimiento de criterios para la educación y sensibilidad de todos los actores, con los valores culturales, ambientales, tecnológicos y económicos, de la herencia en construcciones con tierra con que se cuenta en diversas regiones geográficas.

Asimismo, con el objeto de acometer las intervenciones más adecuadas cuando se 
Agradecimientos

Los autores de este artículo quieren agradecer a la Agencia Española de Cooperación Internacional para el Desarrollo (AECID) la financiación otorgada para el desarrollo del proyecto de referencia $\mathrm{A} / 8378 / 07$, en virtud del cual se ha llevado a cabo el mismo.

Asimismo desean agradecer al personal del Vicerrectorado de Internacionalización y Cooperación al Desarrollo de la Universidad de Oviedo su incondicional apoyo y ayuda económica.

Del mismo modo agradecen al conjunto de personas de la Universidad Central de Las Villas que, cada una dentro de sus posibilidades, han permitido el desarrollo de los trabajos necesarios para la culminación de este artículo. presenten lesiones en la obra patrimonial, se deben considerar los tres aspectos siguientes:

- Debe estudiarse de manera separada el comportamiento y estado técnico de las estructuras y materiales de tierra tanto para el revestimiento como para el soporte.

- Aunque complejo por la evolución histórica de los asentamientos, siempre que se analicen materiales de obra deberán realizarse las correlaciones necesarias con su origen en cantera. Esto facilitará la identificación de los agentes patógenos desencadenantes, así como las causas comunes de deterioro.

- Ante la ausencia de normativa específica para el estudio de la tierra como material de construcción en la edificación, se reconoce la utilidad de la mecánica de suelos para la experimentación y ensayo de sistemas estructurales, materiales y tratamientos.

\section{CONCLUSIONES}

En este artículo se han identificado una serie de factores y mecanismos de alteración condicionantes de la patología típica en las construcciones con tierra. En esta línea se ha procedido a una clasificación de su alterología. Como resultado de tales se ha desarrollado un ordenamiento de los factores intrínsecos y extrínsecos de alteración, que contribuirá a establecer relaciones acertadas con referencia a los indicadores o atributos de alteración que se identifiquen. También, se desarrollado una clasificación de los indicadores macroscópicos de alteración, que permite identificar los fenómenos de ocurrencia en la superficie y en el soporte, así como la magnitud del daño o alteración en la fábrica gracias a la clasificación por grupos de los distintos indicadores.

La identificación de la tipología de intervención en función de la patología caracterizada, constituye un resultado de suma importancia sobre el que debe continuarse profundizando mediante la experimentación en obras, hasta generalizar estas proposiciones.

\section{BIBLIOGRAFÍA}

(1) Monteagudo Rodríguez, I.: Caracterización y evaluación técnica constructiva de mamposterías y tapias de tierra de los siglos XVII, XVIII y XIX. Estudio en la Habana Intramuros. Tesis presentada en opción al grado científico de Doctora en Ciencias Técnicas. Instituto Superior Politécnico "José Antonio Echeverría", Ciudad de La Habana, 2001.

(2) Dassler, L.; Hinojosa, E. M.; Rainer, L.; Teutonico, J. M.: “CRATerre-EAG, ICCROM - The Project GAIA Research Index. Progress report and future developments". Comunicaciones Terra '93. 7 ma. Conferencia Internacional sobre el Estudio y la Conservación de la Arquitectura de Tierra. (1993), pp. 597-604. DGEMN, Silves.

(3) Saroza Horta, B.: Estudio del Adobe en las condiciones de Cuba. Tesis presentada en opción al grado científico de Doctora en Ciencias Técnicas. Universidad Central "Marta Abreu" de las Villas, 2004.

(4) Rodríguez, M. A.; Saroza, B.: "Identificación de la composición óptima del adobe como material de construcción de una escuela en Cuba". Materiales de Construcción. Vol. 56, 282 Abril-junio (2006), pp. 53-62. Consejo Superior de Investigaciones Científicas, Madrid, España. ISSN: 0465-2746.

(5) ICCRO; CRATerre-EAG; GCl; US/ICOMOS: Adobe '90 Preprint, 6 a Conferencia sobre conservación de la arquitectura de Tierra (1990). The Gtty Conservation Institute (GCI), Las Cruces, Nuevo México.

(6) DGEM: "Comunicaciones Terra '03". $7^{a}$ Conferencia Internacional sobre el estudio y conservación de la arquitectura de Teirar (1993). DGEMN, Silves.

(7) Odul, P.: "Pathologie humide de constructions en terre: méthodologie de diagnostic". Adobe '90 Preprint, 6ta. Conferencia Internacional sobre la Conservación de la Arquitectura de Tierra (1990), pp. 404-416. The Getty Conservation Institute (GCI), Las Cruces,Nuevo México.

(8) Monjo, J.; Maldonado, L.; Rohmer, E.; Vela, F.: "Técnicas de intervención para la recuperación y conservación de los muros de tapial en el patrimonio arquitectónico de la Comunidad Autónoma de Madrid". Comunicaciones del III Congreso de Rehabilitación del Patrimonio Arquitectónico y Edificación (1996), pp. 344-351. CEDEX - MOPTMA - Universidad de Granada, Granada.

(9) Romeral, J.; Guinea, M.; Rohmer, E.; Salas, J.: "Primeros resultados del trabajo de investigación sobre la tierra como material de construcción en el IETcc.". Informes de la Construcción (1986). Instituto Eduardo Torrojas, Consejo Superior de Investigaciones Científicas, Madrid.

(10) Chiari, G.: "Caracterización del adobe como material de construcción. Técnicas de preservación". El adobe. Simposio Internacional y Curso taller sobre conservación del adobe. (1983). pp. 33-44. ICOMOS / UNESCO / ICCROM, Lima.

(11) Houben, H.; Guillaud, H.: Traité de Cobnstruction en Terre. CRATerre-EAG, Grenoble, 1989. 